\title{
Candida in Lung Specimens from Non-Neutropenic ICU Patients: Infection or Colonization?
}

\author{
E. Azoulay and B. Schlemmer
}

\section{Introduction}

Candida is an opportunistic yeast normally found in the oral cavity and gastrointestinal tract. Its ability to multiply and to invade the bloodstream and deep tissues is increasing. This has produced new clinical patterns of systemic disease whose expression is dependent on the immune status of the host. Thus, presence of Candida is intimately linked to the effects of chemotherapy and radiation therapy for cancer, hematological malignancies, human immunodeficiency virus (HIV) infection, malnutrition, corticosteroid therapy, and broad-spectrum antibiotics.

New definitions have been developed for classifying invasive fungal infections in cancer patients and/or bone marrow transplant (BMT) recipients as 'proven', 'probable', or 'possible', with the goal of improving the cross-study comparability of clinical and epidemiological data [1]. A diagnosis of proven invasive candidiasis requires a positive blood culture, a positive culture from a normally sterile site (other than the urine and sinuses), or a histologically positive biopsy specimen.

The 'probable' and 'possible' categories are defined based on three groups of criteria:

I host factors: duration of neutropenia longer than 10 days, persistent fever despite broad-spectrum antibiotic therapy, recent use of immunosuppressants, history of definite or probable candidiasis, acquired immunodeficiency syndromes (AIDS), clinical evidence of graft-versus-host disease;

I microbiological criteria: positive blood culture, positive urine culture in a patient without a urinary catheter;

1 Clinical criteria indicating lower respiratory tract infection, sinonasal infection or central nervous system (CNS) infection, with major and minor criteria; criteria for disseminated infection (extensive skin lesions, enophthalmos) or chronic infection (abscesses of the liver or spleen, alkaline phosphatase elevation).

'Probable' invasive candidiasis is defined as the combination of one host factor, one microbiological factor, and either one major or two minor clinical factors. 'Possible' invasive candidiasis is the presence of one host factor and either one major or two minor clinical factors. A positive blood culture should be interpreted according to host factors, neutrophil counts, whether the patient has a central line, and whether the blood was sampled from a peripheral vein or a catheter.

Many intensive care unit (ICU) patients have pulmonary specimens containing Candida counts above the significant 'thresholds' validated for distinguishing colonization from nosocomial pneumonia [2]. Candida colonization in ICU patients reflects 
acquired immunodepression with alterations in both neutrophil function (killing of bacteria, production of oxidants) and alveolar macrophage function [3-5].

We will focus on the interpretation of specimens yielding Candida in non-neutropenic ICU patients. After reviewing the clinical and experimental literature, we will critically appraise this issue, taking care to separate the questions that have been answered from those that remain open. Finally, we will suggest a research agenda for improving our understanding of the continuum that extends from colonization to infection of the lower respiratory tract by Candida in non-neutropenic ICU patients.

\section{- Candida Infection in the ICU: Epidemiological Studies}

We will consider two groups of epidemiological studies, those addressing nosocomial pneumonia in general and those focusing specifically on fungal infections in the ICU. In the former, an approach similar to the standard diagnostic strategy for bacterial nosocomial pneumonia revealed a high prevalence of respiratory tract specimens yielding Candida in concentrations above the threshold used to distinguish colonization from infection. The latter used a more specific approach to the diagnosis of Candida pneumonia and found that the incidence of this condition, although low, is on the rise.

The European Prevalence of Infection in Intensive Care (EPIC) study, a 1995 prevalence survey of nosocomial infection in European ICUs, found that pneumonia contributed $46.9 \%$ of all ICU-acquired infections and that $17 \%$ of ICU-acquired infections were related to yeasts, primarily Candida [2]. The National Nosocomial Infections Surveillance (NNIS) system in the United States showed that Candida species were responsible for $10.1 \%$ of all ICU-acquired infections between 1980 and 1990 [6]. Furthermore, the incidence of Candida infection in hospital patients increased steadily over the study period, 5-fold for candidemia, 2-fold for urinary tract infections, and 1.6-fold for pneumonia [6]. Other studies have reported nosocomial Candida pneumonia in patient subgroups sharing factors such as ICU admission or postoperative status [7-9]. Candida pneumonia has also been described in patients with diabetes mellitus or alcohol abuse; pharyngeal colonization by yeasts is also common in these patients $[10,11]$.

In a study of fungal pneumonia conducted at the National Taiwan University Hospital, a 27-fold increase in nosocomial candidemia occurred between 1980 and 1994 [12]. The diagnosis of fungal pneumonia was based on examination of lung biopsies obtained by thoracotomy or thoracoscopy, percutaneous pleural or lung biopsies obtained under ultrasound guidance, or transbronchial biopsies. The number of cases of fungal pneumonia increased from 5 in 1988 to 30 in 1997. Over the 10 -year study period, 140 cases of fungal pneumonia were identified, including 20 due to Candida. Candida was associated with $70 \%$ mortality, the highest rate among causes of fungal pneumonia; mortality was $40 \%$ for Aspergillus and $6.7 \%$ for Cryptococcus.

In patients with cancer, neutropenia, anticancer chemotherapy, hematological malignancies, or organ transplants, Candida pneumonia is a marker for severe immune system impairment accompanied with selective vulnerability to opportunistic infections [13-15]. In most patients, pneumonia occurs as a metastatic complication of candidemia, often at the terminal stage of a malignant disease with pro- 
found immunodepression [16]. Positive specimens for Candida in these patients raise specific issues, which will not be discussed here.

\section{Candida Colonization: Considerations Specific to ICU Patients}

Studies in ICU and surgery patients have confirmed that a continuum exists from colonization to infection with Candida: colonization is an independent risk factor for systemic candidiasis [17-20]. Pittet et al. found that Candida colonization was an independent risk factor for deep-seated candidal infection and that routine serial testing for colonization at multiple sites (trachea, urine, skin, stool, surgical wounds, and drainage fluids) can be used to define a colonization index (number of positive sites/number of tested sites) [8]. A colonization index greater than 0.5 was associated with an increased risk of deep-seated candidal infection. This risk increase may, in theory, indicate systemic antifungal treatment [21]. Evidence from several studies indicates that candidemia carries a poor prognosis and, consequently, requires early diagnosis or prophylactic measures [19, 22-25]. The beneficial effects of prophylactic treatment have been documented in hematology patients [26-28] and suggested by several ICU studies [29-32]. Work is ongoing to validate pre-emptive antifungal treatment in patients with Candida colonization.

At the respiratory tract, the colonization/infection dichotomy is supported by evidence from autopsy studies (which are older) and clinical studies. Three autopsy studies in 91 cancer patients led to the same conclusions. In immunocompromised patients with systemic candidiasis and pulmonary manifestations, lung pathology consists of necrotizing vasculitis, where the vessels are lined with pus cells containing yeasts. In contrast, in patients without systemic candidiasis, the pulmonary lesions are more likely to consist of pneumonia with intra-alveolar involvement but no vasculitis $[16,33,34]$. Thus, candidal 'pneumonia' seems to exist as two variants. One is secondary to hematogenous dissemination with selective tropism for the blood vessels; this is probably true pulmonary candidiasis. In the other variant, Candida colonizing the oropharynx and gastrointestinal tract spreads along the respiratory tract, ultimately filling the alveoli, so that endobronchial specimens are positive but no clinical or pathological evidence of pneumonia is detectable (Fig. 1) $[35,36]$.

Clinical studies consistently support this distinction. We will focus on two studies that investigated the clinical relevance of 'positive' tracheal or protected distal specimens, bronchoalveolar lavage (BAL) fluid, or bronchial or transbronchial biopsies. Both studies included ICU patients who received mechanical ventilation for longer than 3 days $[37,38]$ and had no evidence of systemic candidiasis. In most patients, lung biopsies or lung autopsy specimens found tracheobronchial colonization without evidence of invasive candidiasis despite positive respiratory specimens. Thus, the usual diagnostic criteria for nosocomial pneumonia do not seem valid for pulmonary candidiasis. Among clinical studies, we will discuss the immediate postmortem study by El-Ebiary et al. [37] in 25 non-neutropenic ICU patients who received mechanical ventilation for longer than 72 hours and died in the ICU. The objective was to investigate correlations between qualitative or quantitative cultures positive for Candida and a diagnosis of pulmonary candidiasis. Immediately after death, endotracheal aspirate, protected specimen brush (PSB), BAL, blind transbronchial biopsies, and bronchoscopically guided biopsies were performed. Ten 


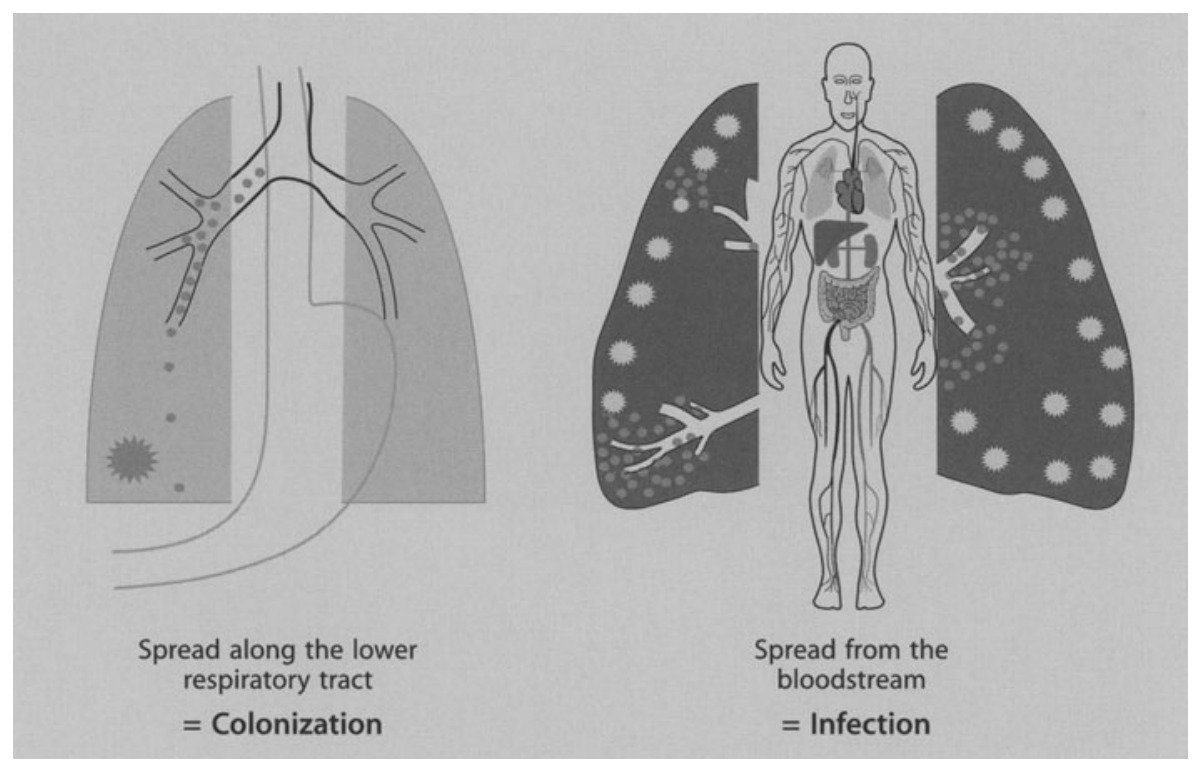

Fig. 1. Pathophysiological concept of Candida pneumonia. Candida pneumonia seems to exist as two variants. Even when Candida concentrations in respiratory specimens are greater than the cuts-off separating colonization from infection, the most likely diagnosis is lower respiratory tract colonization related to spread of Candida colonization from the digestive tract to the tracheobronchial tree [58]. Histology shows no evidence of infectious vasculitis related to Candida invasion. Conversely, when candidemia is suspected, pulmonary involvement can reflect invasive candidiasis of the lungs secondary to hematogenous dissemination of the organism

(40\%) of the 25 patients had at least one biopsy yielding Candida, and Candida contributed $9 \%$ of isolates from biopsies. However, only two (8\%) of these patients had definite pulmonary candidiasis. Alveolitis was found in several patients, but there was no evidence of a causal relation with Candida since other organisms were usually present also. Furthermore, Candida colonization seemed uniform throughout the tracheobronchial tree. Although the small number of patients is a limitation of this study, the data provide a description of Candida colonization in ventilated ICU patients and emphasize the poor correlation between respiratory samples yielding Candida (colonization) and invasive pulmonary candidiasis.

\section{Experimental Models of Pulmonary Candidiasis}

Host defence mechanisms against Candida albicans have been investigated in several animal models, which are summarized in Table 1.

\section{Knockout Mice for the Interferon Gamma Gene}

Evidence for a key role of phagocytic cells stimulated by interferon (IFN)- $\gamma$ has been obtained in knockout mice for the IFN- $\gamma$ gene. As early as the second day after an intraperitoneal injection of $C$. albicans, a lymphoid infiltrate was seen 


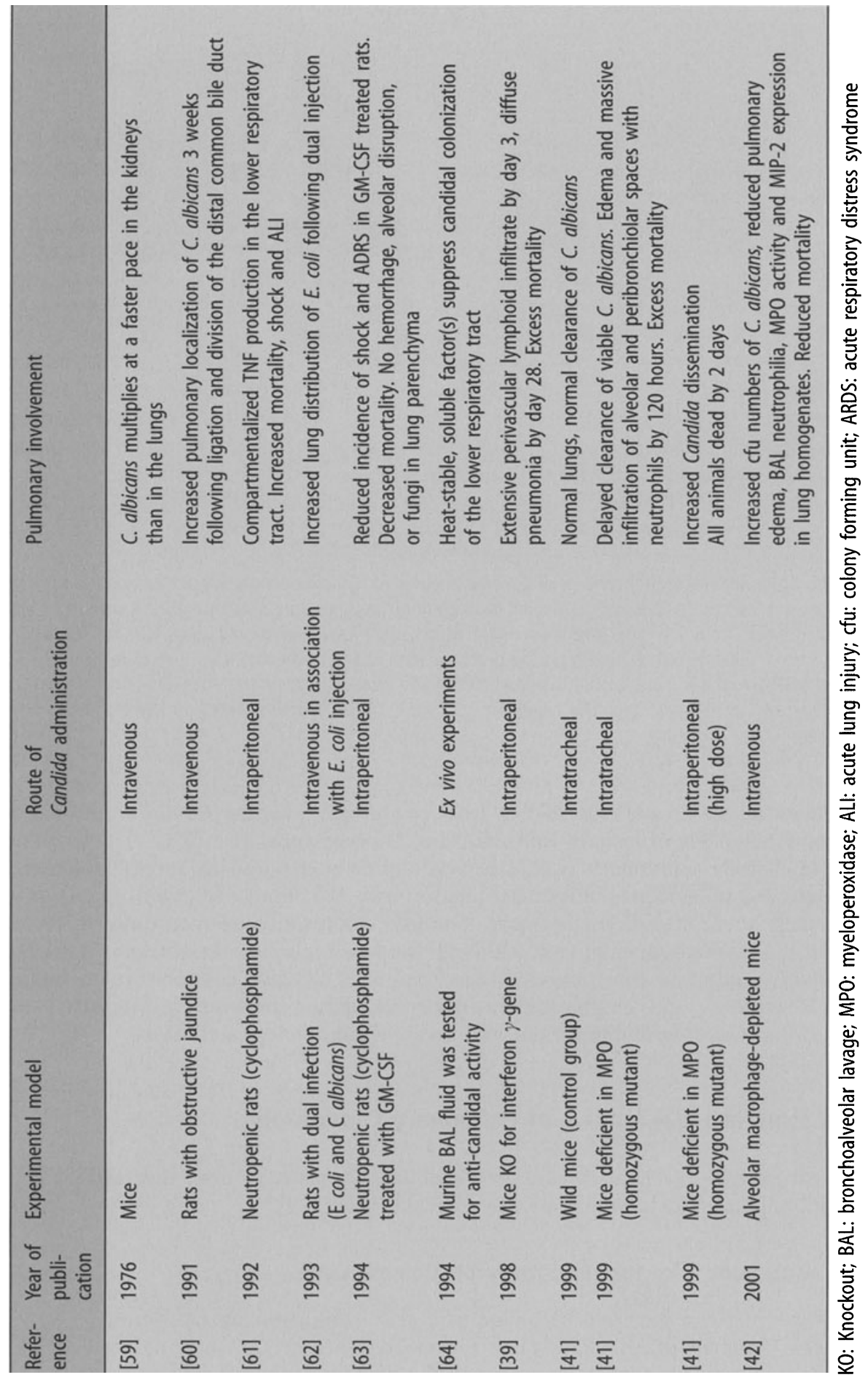


around the pulmonary blood vessels. Subsequently, the infiltrate cleared and diffuse pneumonia developed, without necrotizing vasculitis in the lungs, although necrotizing vasculitis was found in other organs (spleen, liver, heart, and brain). Mortality was considerably higher in the knockout mice than in the controls [39].

\section{Myeloperoxidase-deficient Mice}

Although mononuclear phagocytes are important in combating C. albicans, neutrophils also play a crucial role. Myeloperoxidase is an enzyme found mainly within neutrophils. It converts hydrogen peroxide $\left(\mathrm{H}_{2} \mathrm{O}_{2}\right)$ to hypochlorite $(\mathrm{HOCl})$, a highly cytotoxic compound involved in the neutrophil respiratory burst that releases reactive oxygen species (ROS), which are the key to the antimicrobial effects of neutrophils. Aratani et al. reported increased susceptibility to C. albicans in myeloperoxidase-deficient mice. Although Staphylococcus aureus clearance was normal in these mice, after instillation of C. albicans pneumonia was more likely to develop, and once developed to be fatal $[40,41]$.

\section{Role of the Alveolar Macrophage in Defence Mechanisms against Candida}

Another study established a role for alveolar macrophages in neutrophil recruitment induced by Candida instillation. This role is mediated by the cytokine macrophage inflammatory protein (MIP)-2, which is a chemoattractant for neutrophils. Mice deficient in alveolar macrophages were less likely to die than control mice during candidemia. [42] Yet, Candida concentrations were higher in the deficient mice. Neutrophil counts in BAL fluid were lower in the deficient mice than in the controls. Neutrophil recruitment was related to secretion of MIP-2 by alveolar macrophages [42].

In general, experimental models for invasive candidiasis have been developed only in immunocompromised animals $[43,44]$. Defence mechanisms against Candida albicans and the pathophysiology of systemic candidiasis have been only partly elucidated [45-47]. Phagocyte dysfunction is associated with susceptibility to systemic fungal infections. Phagocytosis of $C$. albicans is optimal after opsonization, which is required for phagocytosis by mononuclear cells [45-47], via a mechanism strongly dependent on nitric oxide (NO) [48]. The pivotal role for dendritic cells in the phagocytosis of $C$. albicans has been established recently [49]. Experimental models have been used to develop agents that are active on $C$. albicans yet have limited toxicity to the patient; efforts have also been made to enhance the antifungal effects of the immune system $[50,51]$. Thus, human recombinant myeloperoxidase, IFN- $\gamma$, or granulocyte/macrophage colony-stimulating factor (GM-CSF) stimulate mononuclear phagocytes and enhance $C$. albicans phagocytosis and killing [50-52]

\section{Conclusion of the Literature Review}

Positive specimens for Candida are common in patients with immunodepression or prolonged broad-spectrum antibiotic therapy [37, 38]. Invasive pulmonary candidiasis is an exceedingly rare entity that seems to represent the pulmonary expression of hematogenous dissemination. It is intimately linked to profound immuno- 
depression, generally systemic (bone marrow failure, chemotherapy, HIV infection) but occasionally local (prolonged intubation in the ICU), and occurring mainly in terminally ill patients with several interlinked conditions (host factors, treatments, risk factors) [16]. Nevertheless, endobronchial specimens yielding Candida in counts above or below positivity thresholds remain clinically relevant: they indicate 'relative' immunodepression, particularly in ICU patients or after surgery (postaggression immunodepression). In this situation, curative antifungal treatment should be discussed. Because a respiratory specimen yielding Candida indicates bronchial colonization, a search for colonization at other sites is in order, to allow estimation of the colonization index, which is known to correlate well with secondary emergence of systemic candidiasis $[8,21,30,31]$.

Although we will not review the details of antifungal treatment, we will point out that treatment decisions depend on patient-related factors, the nature and severity of clinical manifestations, blood culture results, whether bone marrow failure is present, and whether other sites are colonized (colonization index). The recommendations issued by the British Society for Antimicrobial Chemotherapy Working Party can be used to select the antifungal agent [53, 54]; however, it should be borne in mind that the experts who developed these recommendations did not include the lungs among the targets of deep-seated candidal infection.

\section{Suggested Research Agenda}

Below is a suggested research agenda designed to help clinicians interpret pulmonary specimens yielding Candida in non-neutropenic ICU patients.

\section{Epidemiological Questions}

- What is the epidemiology of lower respiratory tract colonization by Candida in the ICU (host factors, Candida species involved)?

- What are the risk factors for acquiring Candida colonization of the lower respiratory tract? Identification of these risk factors would define a patient subset likely to benefit from prophylactic antifungal treatment strategies.

\section{Impact of Lower Respiratory Tract Colonization by Candida in ICU Patients}

What are the morbidity and mortality attributable to Candida colonization of the lower respiratory tract?

I Is it possible to create, under experimental conditions, a model of invasive systemic candidiasis developing from lower respiratory tract Candida colonization in immunocompetent animals?

- How specific is lower respiratory tract Candida colonization? Does colonization at this site provide additional information over colonization at other sites? Does it have specific meaning in mechanically ventilated patients, given the described risk of translocation of bacteria colonizing the lower respiratory tract [55-57]? 


\section{Does Lower Respiratory Tract Candida Colonization per se Indicate Pre-emptive Antifungal Treatment in Patients on Mechanical Ventilation?}

The value of pre-emptive or preventive treatment has been established in high-risk ICU patients [30] and is being evaluated in patients with multiple-site Candida colonization. The problem here is to discuss pre-emptive antifungal treatment in nonneutropenic patients with lung specimens yielding Candida, adjusting on the colonization index. The variable of interest will not necessarily be the mortality rate or incidence of candidemia; treatment efficacy could be evaluated based on criteria such as morbidity (ICU length of stay, mechanical ventilation duration), acquisition of bacteria with multiple drug resistance, emergence of resistance to the antifungal agent used, or selection of resistant Candida species.

\section{References}

1. Ascioglu S, Rex JH, de Pauw B, et al (2002) Defining opportunistic invasive fungal infections in immunocompromised patients with cancer and hematopoietic stem cell transplants: an international consensus. Clin Infect Dis 34:7-14

2. Vincent JL, Bihari DJ, Suter PM, et al (1995) The prevalence of nosocomial infection in intensive care units in Europe. Results of the European Prevalence of Infection in Intensive Care (EPIC) Study. EPIC International Advisory Committee. JAMA 274:639-644

3. Stephan F, Yang K, Tankovic J, et al (2002) Impairment of polymorphonuclear neutrophil functions precedes nosocomial infections in critically ill patients. Crit Care Med 30:315322

4. Nakos G, Malamou-Mitsi VD, Lachana A, et al (2002) Immunoparalysis in patients with severe trauma and the effect of inhaled interferon-gamma. Crit Care Med 30:1488-1494

5. Kox WJ, Volk T, Kox SN, Volk HD (2000) Immunomodulatory therapies in sepsis. Intensive Care Med 26:S124-128

6. Jarvis WR (1995) Epidemiology of nosocomial fungal infections, with emphasis on Candida species. Clin Infect Dis 20:1526-1530

7. Eggimann P, Francioli P, Bille J, et al (1999) Fluconazole prophylaxis prevents intra-abdominal candidiasis in high-risk surgical patients. Crit Care Med 27:1066-1072

8. Pittet D, Monod M, Suter PM, Frenk E, Auckenthaler R (1994) Candida colonization and subsequent infections in critically ill surgical patients. Ann Surg 220:751-758

9. Vincent JL, Anaissie E, Bruining H, et al (1998) Epidemiology, diagnosis and treatment of systemic Candida infection in surgical patients under intensive care. Intensive Care Med 24:206-216

10. Fernandez-Sola J, Junque A, Estruch R, Monforte R, Torres A, Urbano-Marquez A (1995) High alcohol intake as a risk and prognostic factor for community-acquired pneumonia. Arch Intern Med 155:1649-1654

11. Wheat LJ (1980) Infection and diabetes mellitus. Diabetes Care 3:187-197

12. Chen KY, Ko SC, Hsueh PR, Luh KT, Yang PC (2001) Pulmonary fungal infection: emphasis on microbiological spectra, patient outcome, and prognostic factors. Chest 120:177-184

13. von Eiff M, Roos N, Fegeler W, et al (1994) Pulmonary fungal infections in immunocompromised patients: incidence and risk factors. Mycoses 37:329-335

14. von Eiff M, Zuhlsdorf M, Roos N, Hesse M, Schulten R, van de Loo J (1995) Pulmonary fungal infections in patients with hematological malignancies - diagnostic approaches. Ann Hematol 70:135-141

15. Bodey GP (1984) Candidiasis in cancer patients. Am J Med 77:13-19

16. Masur H, Rosen PP, Armstrong D (1977) Pulmonary disease caused by Candida species. Am J Med 63:914-925

17. Wey SB, Mori M, Pfaller MA, Woolson RF, Wenzel RP (1989) Risk factors for hospital-acquired candidemia. A matched case-control study. Arch Intern Med 149:2349-2353

18. Wey SB, Mori M, Pfaller MA, Woolson RF, Wenzel RP (1988) Hospital-acquired candidemia. The attributable mortality and excess length of stay. Arch Intern Med 148:2642-2645 
19. Petri MG, Konig J, Moecke HP, et al (1997) Epidemiology of invasive mycosis in ICU patients: a prospective multicenter study in 435 non-neutropenic patients. Paul-Ehrlich Society for Chemotherapy, Divisions of Mycology and Pneumonia Research. Intensive Care Med 23:317-325

20. Eubanks PJ, de Virgilio C, Klein S, Bongard F (1993) Candida sepsis in surgical patients. Am J Surg 166:617-619

21. Eggimann P, Pittet D (2001) [Candidiasis among non-neutropenic patients: from colonization to infection]. Ann Fr Anesth Reanim 20:382-388

22. Trick WE, Fridkin SK, Edwards JR, Hajjeh RA, Gaynes RP (2002) Secular trend of hospital-acquired candidemia among intensive care unit patients in the United States during 1989-1999. Clin Infect Dis 35:627-630

23. Leleu G, Aegerter P, Guidet B (2002) Systemic candidiasis in intensive care units: A multicenter, matched-cohort study. J Crit Care 17:168-175

24. Blot S, Vandewoude K, Hoste E, Poelaert J, Colardyn F (2001) Outcome in critically ill patients with candidal fungaemia: Candida albicans vs Candida glabrata. J Hosp Infect 47: 308-313

25. Rangel-Frausto MS, Wiblin T, Blumberg HM, et al (1999) National epidemiology of mycoses survey (NEMIS): variations in rates of bloodstream infections due to Candida species in seven surgical intensive care units and six neonatal intensive care units. Clin Infect Dis 29:253-258

26. Goodman JL, Winston DJ, Greenfield RA, et al (1992) A controlled trial of fluconazole to prevent fungal infections in patients undergoing bone marrow transplantation. $\mathrm{N}$ Engl J Med 326:845-851

27. MacMillan ML, Goodman JL, DeFor TE, Weisdorf DJ (2002) Fluconazole to prevent yeast infections in bone marrow transplantation patients: a randomized trial of high versus reduced dose, and determination of the value of maintenance therapy. Am J Med 112:369379

28. Winston DJ, Chandrasekar PH, Lazarus HM, et al (1993) Fluconazole prophylaxis of fungal infections in patients with acute leukemia. Results of a randomized placebo-controlled, double-blind, multicenter trial. Ann Intern Med 118:495-503

29. Pelz RK, Hendrix CW, Swoboda SM, et al (2001) Double-blind placebo-controlled trial of fluconazole to prevent candidal infections in critically ill surgical patients. Ann Surg 233:542-548

30. Garbino J, Lew D, Romand J-A, Hugonnet S, Auckenthaler R, Pittet D (2002) Prevention of severe Candida infections in non-neutropenic, high-risk, critically ill patients. A randomized, double-blind, placebo-controlled trial in patients treated by selective digestive decontamination. Intensive Care Med 28:1708-1717

31. Calandra T, Marchetti O (2003) Antifungal prophylaxis for intensive care unit patients: let's fine tune it. Intensive Care Med (in press)

32. Rex JH, Sobel JD (2001) Prophylactic antifungal therapy in the intensive care unit. Clin Infect Dis 32:1191-1200

33. Haron E, Vartivarian S, Anaissie E, Dekmezian R, Bodey GP (1993) Primary Candida pneumonia. Experience at a large cancer center and review of the literature. Medicine (Baltimore) 72:137-142

34. Rose HD, Sheth NK (1978) Pulmonary candidiasis. A clinical and pathological correlation. Arch Intern Med 138:964-965

35. Azoulay E, Limal N, Mayaud C, Schlemmer B (2001) Bronchial specimens positive to candida: infection or colonization. Réanimation 10:323-328

36. Azoulay E, Mayaud C (1999) [Candida pneumopathy: fact or fiction?]. Rev Pneumol Clin 55:349-351

37. el-Ebiary M, Torres A, Fabregas N, et al (1997) Significance of the isolation of Candida species from respiratory samples in critically ill, non-neutropenic patients. An immediate postmortem histologic study. Am J Respir Crit Care Med 156:583-590

38. Rello J, Esandi ME, Diaz E, Mariscal D, Gallego M, Valles J (1998) The role of Candida sp isolated from bronchoscopic samples in nonneutropenic patients. Chest 114:146-149 
39. Kaposzta R, Tree P, Marodi L, Gordon S (1998) Characteristics of invasive candidiasis in gamma interferon- and interleukin-4-deficient mice: role of macrophages in host defense against Candida albicans. Infect Immun 66:1708-1717

40. Aratani Y, Kura F, Watanabe H, et al (2000) Differential host susceptibility to pulmonary infections with bacteria and fungi in mice deficient in myeloperoxidase. $J$ Infect Dis 182:1276-1279

41. Aratani Y, Koyama H, Nyui S, Suzuki K, Kura F, Maeda N (1999) Severe impairment in early host defense against Candida albicans in mice deficient in myeloperoxidase. Infect Immun 67:1828-1836

42. Kubota Y, Iwasaki Y, Harada H, et al (2001) Role of alveolar macrophages in Candida-induced acute lung injury. Clin Diagn Lab Immunol 8:1258-1262

43. Fulurija A, Ashman RB, Papadimitriou JM (1996) Early inflammatory responses to Candida albicans infection in inbred and complement-deficient mice. FEMS Immunol Med Microbiol 14:83-94

44. Fulurija A, Ashman RB, Papadimitriou JM (1996) Neutrophil depletion increases susceptibility to systemic and vaginal candidiasis in mice, and reveals differences between brain and kidney in mechanisms of host resistance. Microbiology 142:3487-3496

45. Marodi L, Forehand JR, Johnston RB Jr (1991) Mechanisms of host defense against Candida species. II. Biochemical basis for the killing of Candida by mononuclear phagocytes. J Immunol 146:2790-2794

46. Marodi L, Korchak HM, Johnston RB Jr (1991) Mechanisms of host defense against Candida species. I. Phagocytosis by monocytes and monocyte-derived macrophages. J Immunol 146:2783-2789

47. Marodi L (1997) Local and systemic host defense mechanisms against Candida: immunopathology of candidal infections. Pediatr Infect Dis J 16:795-801

48. Blasi E, Pitzurra L, Puliti M, et al (1994) Different events involved in the induction of macrophage tumor necrosis factor by Candida albicans and lipopolysaccharide. Cell Immunol 157:501-509

49. Newman SL, Holly A (2001) Candida albicans is phagocytosed, killed, and processed for antigen presentation by human dendritic cells. Infect Immun 69:6813-6822

50. Marodi L, Tournay C, Kaposzta R, Johnston RB Jr, Moguilevsky N (1998) Augmentation of human macrophage candidacidal capacity by recombinant human myeloperoxidase and granulocyte-macrophage colony-stimulating factor. Infect Immun 66:2750-2754

51. Marodi L, Johnston RB Jr (1993) Enhancement of macrophage candidacidal activity by interferon-gamma. Immunodeficiency 4:181-185

52. Marodi L, Schreiber S, Anderson DC, MacDermott RP, Korchak HM, Johnston RB Jr (1993) Enhancement of macrophage candidacidal activity by interferon-gamma. Increased phagocytosis, killing, and calcium signal mediated by a decreased number of mannose receptors. J Clin Invest 91:2596-2601

53. British Society for Antimicrobial Chemotherapy Working Party (1994) Management of deep Candida infection in surgical and intensive care unit patients. Intensive Care Med 20:522-528

54. Rex JH, Walsh TJ, Sobel JD, et al (2000) Practice guidelines for the treatment of candidiasis. Infectious Diseases Society of America. Clin Infect Dis 30:662-678

55. Cakar N, Akinci O, Tugrul S, et al (2002) Recruitment maneuver: does it promote bacterial translocation? Crit Care Med 30:2103-2106

56. Murphy DB, Cregg N, Tremblay L, et al (2000) Adverse ventilatory strategy causes pulmonary-to-systemic translocation of endotoxin. Am J Respir Crit Care Med 162:27-33

57. Nahum A, Hoyt J, Schmitz L, Moody J, Shapiro R, Marini JJ (1997) Effect of mechanical ventilation strategy on dissemination of intratracheally instilled Escherichia coli in dogs. Crit Care Med 25:1733-1743

58. Torres A, El-Ebiary M, Soler N, Monton C, Fabregas N, Hernandez C (1996) Stomach as a source of colonization of the respiratory tract during mechanical ventilation: association with ventilator-associated pneumonia. Eur Respir J 9:1729-1735

59. Rogers T, Balish E (1976) Experimental Candida albicans infection in conventional mice and germfree rats. Infect Immun 14:33-38 
60. Katz S, Merkel GJ, Folkening WJ, Rosenthal RS, Grosfeld JL (1991) Impaired clearance and organ localization of Candida albicans in obstructive jaundice. J Pediatr Surg 26:904-906

61. Lechner AJ, Tredway TL, Brink DS, Klein CA, Matuschak GM (1992) Differential systemic and intrapulmonary TNF-alpha production in Candida sepsis during immunosuppression. Am J Physiol 263:L526-535

62. Katz S, Merkel GJ, Folkening WJ, Rosenthal RS, Grosfeld JL (1993) Blood clearance and organ localization of Candida albicans and $\mathrm{E}$ coli following dual infection in rats. J Pediatr Surg 28:329-332

63. Lechner AJ, Lamprech KE, Potthoff LH, Tredway TL, Matuschak GM (1994) Recombinant GM-CSF reduces lung injury and mortality during neutropenic Candida sepsis. Am J Physiol 266:L561-568

64. Samaranayake LP, Tobgi RS, MacFarlane TW (1994) Anti-candida activity of murine bronchoalveolar lavage fluid. J Med Microbiol 40:350-357 